\title{
Pregabalin for the management of fibromyalgia syndrome
}

\author{
This article was published in the following Dove Press journal: \\ Journal of Pain Research \\ 19 June 2010 \\ Number of times this article has been viewed
}

\section{Chad S Boomershine \\ Department of Medicine, Vanderbilt University, Nashville, TN, USA}

Correspondence: Chad S Boomershine T32 I 9 MCN, I I6I 2 Ist Ave South, Nashville, TN 37232-268I, USA

Tel + I 6I5 3224746

$\mathrm{Fax}+\mathrm{I} 6153226248$

Email chad.boomershine@vanderbilt.edu
Abstract: This last article in a three-part series on approved medications for managing fibromyalgia syndrome (FMS) reviews pregabalin $\left(\right.$ Lyrica $\left.^{\circledR}\right)$. Pregabalin was the first drug approved for FMS management and, as an anticonvulsant, differs from the other approved agents that are antidepressants. Pregabalin inhibits presynaptic excitatory neurotransmitter release by blocking $\alpha_{2} \delta$ calcium channels. Five randomized, placebo-controlled trials have demonstrated pregabalin reduces pain and improves sleep and health-related quality of life in FMS patients. While indicated dosing is $300-450 \mathrm{mg}$ divided twice daily, initial dosing of 25-50 mg at night is recommended owing to side effects including somnolence, dizziness, and cognitive dysfunction. Since side effects such as weight gain and peripheral edema are dose-related, uptitration in weekly increments based on tolerability and therapeutic response is recommended. Due to its lack of protein binding and negligible hepatic metabolism, pregabalin can be safely combined with other medications and used in patients with renal failure when the dose is appropriate. Pregabalin may worsen sedation when combined with central nervous system depressants. Pregabalin should be discontinued gradually. Pregabalin-treated patients should be monitored for the emergence or worsening of depression, suicidal thoughts or behavior. Pregabalin in combination with the other approved medications may be synergistic in treating FMS.

Keywords: review, Lyrica ${ }^{\circledR}$, treatment, depression

\section{Introduction}

Fibromyalgia syndrome (FMS) is a disorder of chronic widespread pain (CWP) and tender points associated with a number of other symptoms including fatigue, nonrestorative sleep, depression, anxiety, and stiffness. ${ }^{1}$ FMS is a common disorder occurring around the world, with published prevalence rates of up to $5 \%$. $^{2}$ However, these rates likely underestimate the true prevalence of FMS since they are based on criteria that do not lead to the identification of half of clinically diagnosed FMS patients. ${ }^{3}$ FMS is a significant cause of morbidity, resulting in high costs due to health care utilization and disability comparable to those seen in patients with rheumatoid arthritis. ${ }^{4}$ Evidence-based management guidelines recommend a multidisciplinary treatment approach that combines pharmacologic and nonpharmacologic modalities individually tailored according to patient symptoms to effectively manage FMS symptoms..$^{5}$ Nonpharmacologic treatments include graduated aerobic and resistance exercise, education and cognitive-behavioral therapies. There are currently three pharmacologic therapies indicated by the United States (US) Food and Drug Administration (FDA) for the management of FMS: duloxetine $\left(\right.$ Cymbalta $\left.^{\circledR}\right)$, milnacipran $\left(\right.$ Savella $\left.^{\mathrm{TM}}\right)$ and 
pregabalin $\left(\operatorname{Lyrica}^{\circledR}\right)$. Previous articles in this series have reviewed duloxetine and milnacipran. ${ }^{6,7}$ This article will focus on the pharmacology, mechanism of action, efficacy, safety, and tolerability of pregabalin in the hopes it will aid providers in caring for FMS patients.

\section{Review of pharmacology, mode of action and pharmacokinetics of pregabalin}

On June 21, 2007, pregabalin became the first medication to receive FDA approval for the management of FMS. Pregabalin had previously been approved for treating diabetic peripheral neuropathic pain (DPNP), post-herpetic neuralgia (PHN), and as adjunctive therapy for adult patients with partial onset seizures. ${ }^{8}$ Pregabalin differs from the other two indicated FMS medications by the fact that it is classified as an anticonvulsant and not an antidepressant. Pregabalin, and the closely related drug gabapentin $\left(\right.$ Neurontin $\left.^{\circledR}\right)$, is a structural analog of the neurotransmitter $\gamma$-aminobutyric acid (GABA). Pregabalin is an $\alpha_{2} \delta$ calcium channel antagonist that inhibits presynaptic neurotransmitter release. The pharmacologic actions of pregabalin are restricted to neurons, as pregabalin has no effect on blood pressure or heart rate even at high dosages, unlike the vascular calcium channel blockers. While its mechanism of action is not completely understood, the analgesic, anticonvulsant, and anxiolytic actions of pregabalin are thought to result from decreasing central nervous system (CNS) concentrations of excitatory neurotransmitters including glutamate and substance $\mathrm{P}^{9,10}$ FMS patients are known to have increased CNS levels of substance $\mathrm{P}$ and glutamate, ${ }^{11,12}$ and increased levels of these neurotransmitters have been implicated in the process of central pain amplification that is thought to underlie FMS. ${ }^{13}$ Related to its inhibition of nerve signaling, pregabalin can have sedative effects. Because of this, use of pregabalin with other CNS depressants should be limited and patients should be warned not to operate heavy machinery until they determine how pregabalin will affect them.

Pregabalin has numerous favorable pharmacokinetic properties including negligible serum protein binding (limiting the possibility for displacing other drugs), negligible hepatic metabolism, and linear dosing kinetics. ${ }^{8}$ These properties limit drug-drug interactions, making pregabalin amenable to combination therapy, which is important in FMS management since patients commonly require multiple medications to manage their symptoms. Pregabalin has a relatively short half-life (6.3 hours), necessitating twice-daily dosing to maintain plasma drug levels. However, many FMS patients complain of somnolence and dizziness as well as worsening of fatigue and cognitive dysfunction when pregabalin is taken during the day. The short half-life of pregabalin can be exploited to limit side effects during the day by dosing all or most of pregabalin with the evening meal or at bedtime when somnolence can be beneficial for improving sleep. Pregabalin absorption is altered by taking it with food, resulting in prolongation of time to maximum plasma concentration $\left(\mathrm{T}_{\max }\right)$ to 3 hours and decreasing the maximum plasma concentration $\left(\mathrm{C}_{\max }\right)$ by $25 \%-30 \% .{ }^{8}$ Since this can limit side effects, taking pregabalin with food is recommended.

\section{Pregabalin efficacy studies}

A recent meta-analysis that systematically analyzed the scientific literature identified five randomized, double-blind, placebo-controlled trials (RCTs) of pregabalin for the treatment of FMS that met standardized criteria for validity and methodological quality, ${ }^{14}$ making pregabalin the most thoroughly studied of the FDA-approved therapies. All enrolled patients were required to meet American College of Rheumatology classification criteria for FMS and the primary endpoint for all the RCTs was improvement in pain symptoms. ${ }^{1}$ Pain was also the primary endpoint for FMS RCTs of duloxetine, ${ }^{6}$ whereas milnacipran used composite endpoints combining pain with global and physical function improvement. ${ }^{7}$ All pregabalin trials required FMS patients to have baseline visual analog scale (VAS) pain scores of $\geq 4 \mathrm{~cm}$ on a $10-\mathrm{cm}$ scale, consistent with RCTs of duloxetine and milnacipran. Also consistent with duloxetine and milnacipran trials, FMS patients were excluded from pregabalin RCTs if they had evidence of inflammatory rheumatic disease, or clinically significant or unstable medical or psychological conditions. Patients were also excluded if they were applying for disability or engaged in litigation related to FMS. As in the RCTs for the other approved FMS drugs, the majority of patients in the pregabalin trials were middle-aged, Caucasian women, making generalization of trial results to other genders or ethnic groups problematic. All trials also used twice-daily dosing with rapid uptitration of study medication, with patients typically reaching doses of $300 \mathrm{mg} /$ day by the end of the first week and maximal study doses by the end of week 2 . Since such rapid dose titration is not typically used in the clinic, withdrawal and adverse event (AE) rates seen in the RCTs may be higher than those observed clinically.

The first RCT published in 2005 was an 8-week trial that assessed 529 FMS patients equally divided to receive placebo or pregabalin at doses of 150,300 , and $450 \mathrm{mg}$ divided twice daily. ${ }^{15}$ Only the $450-\mathrm{mg}$ /day group met the primary 
study endpoint, with significantly lower pain scores averaged over the last week $(-0.93$ on a $0-10$ scale, $P \leq 0.001)$ and significantly more patients with either a $\geq 30 \%$ or $\geq 50 \%$ improvement in baseline pain scores compared with placebo (48.4\% vs $27.1 \%, P=0.003$ and $29 \%$ vs $13 \%, P=0.003$, respectively). It should be noted that a $\geq 30 \%$ improvement is considered the minimally clinically significant difference that can be measured, whereas a $\geq 50 \%$ improvement is considered a better measure of an improvement that is meaningful in the daily life of patients. ${ }^{16}$ Pregabalin treatment at $300 \mathrm{mg} /$ day or $450 \mathrm{mg} /$ day was associated with statistically significant improvements in sleep quality, fatigue, and global impression of change (both patient and clinician reported). However, it should be noted that this trial used last observation carried forward (LOCF) to analyze the data. While a typical analytic method for RCTs, LOCF is a less conservative analysis than baseline observation carried forward (BOCF), which is required by the FDA for pain studies. LOCF allows the last data collected from patients who do not complete a trial to be used as endpoint data, whereas BOCF requires baseline data be used. This means that drug effects on noncompleting patients are included in analyses using LOCF but not in analyses using BOCF. Pregabalin effects were rapid, with improvements seen as early as the first week of treatment.

The second RCT published in March 2008 was a 13-week trial that assessed 748 FMS patients. ${ }^{17}$ Patients were randomized equally to receive either placebo or pregabalin at doses of 300,450 , or $600 \mathrm{mg}$ divided twice daily. In addition to the primary endpoint evaluating pain improvement, the trial had a co-primary endpoint to evaluate pregabalin efficacy in managing global FMS symptoms by assessing improvement in the Patient Global Impression of Change (PGIC) and the Fibromyalgia Impact Questionnaire (FIQ) total score. The PGIC is a standard patient self-report questionnaire that measures change in patients' overall status after drug treatment on a numeric rating scale ranging from 1 = 'very much improved' to $7=$ 'very much worse', with $4=$ 'no change.' ${ }^{18}$ The FIQ is a 20 -item patient self-report instrument that quantifies the global impact of FMS by querying physical functioning, pain, fatigue, stiffness, morning tiredness, difficulty working (including housework), number of days patients 'felt good', and symptoms of depression and anxiety in the past week. ${ }^{19}$ The FIQ yields a score from 0 to 100 , with higher scores indicating more severe FMS. The second RCT met its primary pain endpoint, with all pregabalin treatment groups having statistically significant improvement in pain compared to placebo-treated groups. While patients in pregabalin-treated groups had significantly greater improvement in PGIC scores compared to those receiving the placebo, the study failed to reach both co-primary endpoints since none of the pregabalin groups had significant improvement in FIQ total scores compared to placebo groups. As in the previously discussed RCT, all three pregabalin doses (300, 450, and $600 \mathrm{mg} /$ day) were associated with statistically significant improvement in sleep quality. However, no statistically significant improvement was seen in symptoms of fatigue as in the previous trial.

The third FMS pregabalin trial, published in June 2008, was a 6-month trial that evaluated the efficacy and durability of pregabalin treatment of FMS pain..$^{20}$ This RCT was conducted in a much different manner than all previous and subsequent FMS trials to date. In this trial, 1051 FMS patients were first assigned to a 6-week open-label pregabalin-treatment period divided into an initial 3-week dose optimization phase followed by a 3 -week fixed-dose phase. Upon entering the dose optimization phase, an open-label baseline assessment was performed to quantify symptoms of pain (using a VAS) and fatigue (using the Multidimensional Assessment of Fatigue, MAF) ${ }^{21}$ sleep quality (using the Medical Outcomes Study (MOS)-sleep scale), ${ }^{22}$ and overall health status (using the FIQ and the MOS 36-item short form survey instrument, SF-36). ${ }^{19,23}$ All FMS patients were then treated with pregabalin at $150 \mathrm{mg} /$ day divided twice daily for 3 days. After 3 days, drug tolerability was assessed. If tolerated, pregabalin dose was increased to $300 \mathrm{mg}$ /day divided twice daily. If patients did not tolerate the drug, they were withdrawn from the trial. At the end of the first week, patients were assessed for efficacy and tolerability. Efficacy was determined by two criteria: 1) $\geq 50 \%$ reduction in pain VAS score from baseline and 2) self-rating of 'much improved' or 'very much improved' on the PGIC scale. If patients showed efficacy and tolerability, dosage was maintained at $300 \mathrm{mg} /$ day throughout the end of the open-label phase. If patients failed to show efficacy but maintained tolerability, pregabalin dose was increased to 450 $\mathrm{mg}$ /day for another week after which they were again assessed for efficacy and tolerability. Patients who then met efficacy and tolerability criteria were maintained on a $450-\mathrm{mg} /$ day dose throughout the end of the open-label phase. Patients who did not meet efficacy but maintained tolerability had their dose increased to $600 \mathrm{mg} /$ day. After 1 week, patients on $600 \mathrm{mg} /$ day were again assessed for efficacy and tolerability and those who met efficacy and tolerability criteria were maintained on that dose throughout the open-label phase. Patients who did not tolerate drug were withdrawn from the trial. By the end of week 3, all patients remaining in the trial were being treated with pregabalin at doses of 300,450 , or $600 \mathrm{mg} /$ day. At this point, patients entered 
a 3-week fixed-dose phase in which they were maintained on stable doses of pregabalin and were assessed each week for the drug's efficacy. To be considered responders and enter the double-blind phase of the trial, patients had to meet both pain and PGIC efficacy criteria at week 4 or 5 and also at week 6 , and had to maintain drug tolerance. For the 6-month double-blind phase, half of responders in each dose group (300, 450, or $600 \mathrm{mg} /$ day) were randomized to either continue pregabalin treatment at the same dose or receive treatment with placebo (after a 2-6 day taper depending on dosage group). All participants were then assessed at week 8 and every 4 weeks thereafter to evaluate efficacy using the pain VAS, MAF, MOS-Sleep Scale, FIQ, and SF-36. The primary outcome of the study was time to loss of therapeutic response (LTR) defined as either a worsening of pain VAS score to within $30 \%$ of the open-labeled baseline score at two consecutive visits in the double-blind phase (with the first of the two visits defining the time to LTR and the second used only for confirmation of LTR) or worsening of FMS symptoms that, in the judgment of the investigator, necessitated alternate treatment. Secondary endpoints compared the difference in time to loss of response for other improved therapeutic outcomes as determined by a change in questionnaire scores indicating worsening of more than the minimally important clinical difference (for the FIQ, MAF, MOS-Sleep Scale, and SF-36) or a score indicating less improvement than 'much improved' on the PGIC. The trial met its primary endpoint, with the time to LTR significantly longer for pregabalin versus placebo treatment $(P<0.0001)$. By day 19 , half of the placebo group had LTR, whereas by the end of the trial more than half of the pregabalin group still had not lost response. At the end of the 6 month double-blind phase, $68 \%$ of pregabalin-treated patients had maintained their therapeutic response compared to $39 \%$ of those treated with the placebo. When the individual pregabalin fixeddosage groups of 300,450 , and $600 \mathrm{mg}$ /day were compared with their corresponding placebo groups, each pregabalin treatment group was associated with a significantly longer time to LRT. All secondary efficacy endpoints also demonstrated significantly greater time to LTR for pregabalin compared to placebo treatment $(P<0.0001$ for all), with median time to loss of response longer for pregabalin treatment as measured by the PGIC (126 vs 20 days), the FIQ (19 vs 14 days), the MOS-Sleep Scale (42 vs 14 days), the MAF (119 vs 27 days), and SF-36 component scores for physical (49 vs 15 days) and mental (42 vs 14 days) function.
The fourth pregabalin FMS trial was a 14-week RTC that studied 745 patients, and was published in September $2008 .{ }^{24}$ This trial has been criticized because it was preceded by a 1 -week placebo run-in phase that excluded placebo responders, defined as patients with $\geq 30 \%$ decreases in VAS pain scores. ${ }^{25}$ However, trial results are felt by the author to be valid since exclusions occurred prior to randomization, few patients were excluded (19 total), and the results of the trial were accepted by the FDA in support of the FMS indication. As in the above-mentioned 13-week study, the primary outcome variable was improvement in endpoint mean VAS pain scores, with additional primary efficacy parameters including PGIC and FIQ total scores. Patients were randomized to receive placebo or pregabalin at doses of 300,450 , or $600 \mathrm{mg} /$ day divided twice daily. The fourth study met its primary endpoint, with all three pregabalin doses providing significantly greater improvement in endpoint pain scores compared to placebo. The proportion of patients with $\geq 30 \%$ or $\geq 50 \%$ decreases in mean pain scores from baseline to endpoint were significantly greater in all active treatment groups compared with placebo group patients. The $\geq 30 \%$ responder rate was $30 \%$ in the placebo group, $42 \%$ in the $300-\mathrm{mg} /$ day, $50 \%$ in the $450-\mathrm{mg} /$ day, and $48 \%$ in the $600-\mathrm{mg} /$ day pregabalin groups $(P=0.0172, P=0.0002$, and $P=0.0006$, respectively), whereas the $\geq 50 \%$ responder rate was $15 \%$ in the placebo group, $24 \%$ in the $300-\mathrm{mg} /$ day, $27 \%$ for $450-\mathrm{mg} /$ day, and $30 \%$ for $600-\mathrm{mg} /$ day pregalin groups ( $P=0.0372, P=0.0038, P=0.001$, respectively). Contrary to the previous 13 -week RCT, significantly greater improvement for both FIQ and PGIC scores were observed across all pregabalin treatment groups compared to the placebo groups, with the exception of the FIQ score in the 300-mg/day pregabalin group. Consistent with previous trials, all pregabalin doses improved sleep quality but no improvements were seen in fatigue, depression, or anxiety symptoms at indicated doses.

The fifth pregabalin FMS RCT has not been published. However, a description and results are available online and were included as part of a meta-analysis that reviewed it and the other four pregabalin FMS RCTs from which trial information can be obtained. ${ }^{14,26}$ This RCT was a standard 14-week parallel-group trial that randomized 747 FMS patients to treatment with placebo or pregabalin at doses of 300, 450, and 600 $\mathrm{mg}$ /day divided twice daily. The primary endpoint measure was the endpoint mean pain score derived from the subject's daily pain diary, and a co-primary endpoint was PGIC score. Secondary endpoints included evaluations of the efficacy of pregabalin compared with placebo for improvement of 
sleep, function, fatigue, health-related quality of life, and mood disturbance as determined by changes in scores on the MOS-Sleep Scale, FIQ, MAF, SF-36, and the Hospital Anxiety and Depression Scale (HADS) ${ }^{27}$ respectively. As in the fourth study, this trial included a 1-week placebo run-in phase to exclude placebo responders. In contrast to the other studies that were conducted exclusively within the US, this trial was conducted exclusively outside the US at 73 centers in North America (Canada and Mexico), South America (Venezuela), Europe (Denmark, France, Germany, Italy, Netherlands, Portugal, Spain, Sweden, Switzerland, and United Kingdom), Asia (India and Korea) and Australia, indicating that its results may be more broadly applicable. While the primary trial endpoint was reached, only the 450-mg/ day treatment group experienced a statistically significant improvement in the endpoint mean pain score compared with placebo-treated subjects $(P=0.0132)$. As in previous trials, pregabalin-treated patients had significant improvement in sleep quality (all doses) and PGIC scores (450- and 600-mg/ day groups). Pregabalin at $450 \mathrm{mg} /$ day was superior compared with placebo on the FIQ total score, and pregabalin at 450 and $600 \mathrm{mg}$ /day significantly improved SF-36 mental component scores. There were no statistically significant differences for the comparisons between pregabalin and placebo for MAF (fatigue) or HADS (anxiety and depression) scores.

Two recent meta-analyses have assessed the efficacy of pregabalin by analyzing the results from the five FMS trials described above. ${ }^{14,28}$ Both meta-analyses found that pregabalin treatment significantly reduced pain, improved sleep, and improved health-related quality of life in FMS patients over placebo. There were also statistically significant, but less robust, effects of pregabalin on reducing fatigue and anxiety symptoms, but no evidence was seen for improvement in depression symptoms. While the magnitude of beneficial effects was small, with effect sizes ranging from approximately 0.2 to 0.4 using Cohen's categories, these effect sizes are similar to those seen for other analgesic medications felt to be effective in treating chronic pain conditions such as paracetamol or nonsteroidal anti-inflammatory drugs for treating osteoarthritis. ${ }^{29}$ Also, the therapeutic effect of pregabalin on pain was similar to that seen for other FMS treatments such as duloxetine ${ }^{30}$ and the combination of tramadol and acetaminophen..$^{31}$ (While milnacipran likely has similar efficacy in treating FMS pain, there are currently no meta-analyses from which to obtain effect size data.) However, FMS patients typically experience numerous symptoms in addition to pain that can be equally or even more debilitating, and evidence-based FMS management guidelines recommend individualizing therapy based on associated symptoms to optimally manage FMS. ${ }^{5}$ Based on RCTs and clinical experience, pregabalin has been recommended for initial treatment of FMS patients with significant sleep complaints. ${ }^{32}$ While pregabalin may benefit FMS patients with anxiety, these patients should also be screened for depression, since anxiety and depression frequently coexist and pregabalin does not improve depressive symptoms. In FMS patients with coexisting anxiety and depression, duloxetine would likely be a better initial choice since it is indicated for treating all three conditions. ${ }^{33}$ However, FMS patients can be highly variable in the medications to which they respond and treatment recommendations should be considered rough guides rather than absolute rules.

\section{Safety and tolerability of pregabalin}

The best source for information on the safety and tolerability of pregabalin in FMS patients comes from two metaanalyses. ${ }^{14,28} \mathrm{~A}$ large percentage of patients in the trials had at least one $\mathrm{AE}$ regardless of treatment $(74 \%$ and $88 \%$ for placebo and pregabalin, respectively). However, few patients experienced a serious AE (2\%), and serious AE rates did not differ between placebo and pregabalin treatment groups, supporting the safety of pregabalin treatment in FMS patients. Tolerability can be gauged by looking at the discontinuation rates observed in the trials. For the indicated FMS doses, no significant differences were seen in total discontinuation rates between placebo and pregabalin treatments. However, for discontinuations due to an $\mathrm{AE}$, a dose-response relationship was found for pregabalin. While the risk was similar between placebo and pregabalin at $150 \mathrm{mg} /$ day, relative risk for discontinuation was higher for pregabalin compared to placebo for all other doses and increased with dose (1.6, 1.9, and 2.5 for 300,450 , and $600 \mathrm{mg} /$ day, respectively). This is consistent with clinical observations that lower pregabalin doses are better tolerated, and use of the smallest effective dose is recommended.

Dizziness was the most common AE in the FMS trials, and all pregabalin doses were associated with increased relative risk for dizziness compared to placebo. A dose response relationship was seen for dizziness rates, with $11 \%$ for placebo, $23 \%$ for $150 \mathrm{mg} /$ day, $33 \%$ for $300 \mathrm{mg} /$ day, $43 \%$ for $450 \mathrm{mg} /$ day, and $47 \%$ for $600 \mathrm{mg} /$ day of pregabalin. Somnolence was the second most common AE, with a $5 \%$ rate for placebo and rates of $20 \%-23 \%$ for pregabalin doses of $150-600 \mathrm{mg} /$ day. Pregabalin use was also associated with a twofold increased risk for gaining more than 7\% 
in body weight, and this risk occurred regardless of dose. While pregabalin use was also associated with an increased peripheral edema rate $(6 \%$ for indicated pregabalin doses vs $2 \%$ for placebo), edema occurred independent of weight gain. Pregabalin-treated patients also had higher rates of fatigue (averaging 7.6\% for indicated pregabalin doses vs 3.5\% for placebo) and cognitive dysfunction ( $6 \%$ for indicated pregabalin doses vs $1 \%$ for placebo). To limit occurrence of all these AEs, pregabalin dosing primarily at bedtime, with a smaller dose taken on awakening only if needed for symptom control during the day, is recommended. Taking pregabalin with food may also limit AEs by decreasing the $\mathrm{C}_{\max }$ and increasing the $\mathrm{T}_{\max }{ }^{8}$ Since nausea is a frequent $\mathrm{AE}$ seen in FMS patients treated with duloxetine and milnacipran, ${ }^{33,34}$ it is important to realize pregabalin-treated patients had no increase in nausea rates compared to placebo-treated patients ( $9 \%$ vs $8 \%$, respectively), making pregabalin the best choice for FMS patients with gastrointestinal complaints.

All antiepileptic drugs (AED), including pregabalin, warn in their prescribing information that use may be associated with increased suicide risk. The concern for increased suicide risk comes from a pooled analysis of 199 placebo-controlled clinical trials of 11 different AEDs involving 43,892 patients aged 5 years and older conducted by the FDA. ${ }^{35}$ The analysis showed that patients treated with AEDs had approximately twice the risk of suicidal thinking or behavior compared to patients randomized to placebo, with an odds ratio (OR) of $1.80(95 \%$ confidence interval [CI]: 1.24, 2.66). However, only two drugs, topiramate and lamotrigine, had OR-associated confidence intervals that did not cross 1.0 and were therefore associated with a statistically significant increased risk. Also, statistically significant suicide risk with AED treatment was only seen in epileptic patients (OR 3.53, 95\% CI: 1.28, 12.10). Neither psychiatric patients (OR 1.51, 95\% CI: 0.95, 2.45) nor other patient groups (OR 1.87, 95\% CI: 0.81, 4.76) treated with AEDs had statistically significant increased suicide risk. While suicide risk may be increased by AED treatment, the FDA did not feel the risk was sufficient to warrant a black boxed warning in the prescribing information of pregabalin. ${ }^{8}$ This indicates the risk for suicidality with pregabalin treatment of nonepileptic FMS patients is likely to be low. However, since the risk to individual patients cannot be predicted, it is recommended that patients treated with an AED for any indication be monitored for the emergence or worsening of depression, suicidal thoughts or behavior, and/or any unusual changes in mood or behavior. It is important to note that both duloxetine and milnacipran have black box warnings for suicidalilty in their prescribing information. ${ }^{33,34}$

\section{Pregabalin's place in FMS treatment}

Owing to the heterogeneity of FMS patients, an individualized treatment regimen based on associated symptoms is recommended to optimize management. ${ }^{5}$ The FIBRO mnemonic can be used to recall commonly associated FMS symptoms and individualize treatment; ' $\mathrm{F}$ ' for fatigue and fibrofog (cognitive dysfunction), 'I' for insomnia (poor sleep), ' $\mathrm{B}$ ' for blues (depression and/or anxiety), ' $\mathrm{R}$ ' for rigidity (stiffness of muscles and/or joints) and ' $\mathrm{O}$ ' for ow! (pain and work disability). ${ }^{32}$ While all FDA-indicated FMS treatments have similar efficacy in improving pain, meta-analyses of FMS RCTs show pregabalin is unique in its ability to also improve sleep. ${ }^{14,28}$ Pregabalin has been shown to decrease sleep latency and modify sleep architecture by enhancing slow-wave sleep, making it a particularly good choice for FMS patients with sleep complaints. ${ }^{36}$ Pregabalin has also been shown to improve anxiety symptoms in FMS RCT meta-analyses. ${ }^{14,28}$ Analyses of pregabalin treatment in patients with generalized anxiety disorder indicate pregabalin has a moderate effect in treating anxiety symptoms that is comparable to other anxiolytics, including the serotonin and norepinephrine reuptake inhibitors (SNRIs), at doses of $200 \mathrm{mg} /$ day and higher. ${ }^{37,38} \mathrm{FMS}$ patients have a fivefold increased rate of anxiety disorders compared to controls, ${ }^{39}$ indicating the anxiolytic effect of pregabalin may benefit many FMS patients. However, pregabalin does not improve symptoms of depressed mood in FMS patients. ${ }^{14,28}$ Owing to the high concurrence of depressive and anxiety disorders, it is recommended that anxious FMS patients be screened for the presence of depression and, if depressive symptoms are present, duloxetine be considered as first-line since it is indicated to treat major depressive disorder, generalized anxiety disorder, and FMS. ${ }^{33}$ Also, since pregabalin use has been associated with worsening cognitive dysfunction ('fibrofog'), ${ }^{14,28}$ consideration should be given for using milnacipran first-line in FMS patients with baseline cognitive dysfunction since milnacipran can improve cognition. ${ }^{7}$ At appropriate doses, pregabalin can be used in patients with any degree of renal impairment including dialysis patients, making it an excellent choice for FMS patients with renal impairment. ${ }^{8}$ An important difference between pregabalin and the other indicated FMS medications is that pregabalin use is not associated with nausea, making pregabalin an excellent choice for FMS patients with baseline gastrointestinal complaints such as irritable bowel syndrome. Pregabalin use has also not been associated with headache, ${ }^{8}$ making it a better choice for FMS patients with migraine than the other two indicated drugs (duloxetine and milnacipran), which often cause headache. . $^{33,34}$ 
Pregabalin has numerous favorable pharmacokinetic properties including no serum protein binding and negligible hepatic metabolism that make it safe for use in combination with other drugs. ${ }^{8}$ Since the proposed mechanisms of action differ between pregabalin and the other indicated FMS drugs, with pregabalin thought to decrease excitatory nerve signaling and duloxetine and milnacipran thought to increase inhibitor signaling, it is reasonable to hypothesize that combination therapy may be synergistic. A combination trial of the $\alpha_{2} \delta$ calcium channel antagonist gabapentin and the SNRI venlafaxine for individuals with diabetic neuropathy supports this theory. ${ }^{40}$ This trial showed that the combination of gabapentin and venlafaxine improved symptoms of pain, fatigue, mood disturbance, and insomnia in patients with neuropathic pain who had not responded to gabapentin monotherapy previously. The potential for synergy may have significant clinical implications, as currently indicated FMS therapies typically have only mild efficacy in treating FMS symptoms. ${ }^{28,30}$ Clinical experience has shown that the combination of pregabalin at bedtime with duloxetine or milnacipran upon awakening can often provide therapeutic efficacy in FMS patients who have failed monotherapy utilizing lower, often better tolerated, drug doses. Randomized combination trials are needed to better understand the risks and benefits of combination therapy in treating FMS patients. In the interim, combination therapy should be used cautiously owing to the potential for additive adverse effects.

\section{Conclusions and key points}

1. Pregabalin was the first FDA drug approved drug for the management of FMS syndrome.

2. Pregabalin is an $\alpha_{2} \delta$ calcium channel antagonist categorized as an antiepileptic, thought to work by decreasing excitatory nerve signaling.

3. Pregabalin has favorable pharmacokinetic properties including no serum protein binding, negligible hepatic metabolism and linear dosing kinetics.

4. The safety and efficacy of pregabalin in managing FMS have been demonstrated by five RCTs involving over 3800 FMS patients.

5. Pregabalin can reduce symptoms of pain, anxiety, and fatigue, and can improve global function and sleep quality in FMS patients.

6. Adverse events associated with pregabalin use include dizziness, somnolence, weight gain, peripheral edema, fatigue, and cognitive dysfunction.

7. Pregabalin-treated patients should be monitored for the emergence or worsening of depression, suicidal thoughts or behavior, and/or any unusual changes in mood or behavior.

8. The combination of pregabalin at bedtime and either duloxetine or milnacipran on awakening may be synergistic in managing FMS symptoms, but clinical trials using combination therapy are needed to identify potential additive adverse events.

\section{Disclosure}

The author works as a consultant for Eli Lilly and Co., Forest Laboratories Inc., and Pfizer Inc. Dr Boomershine receives research funding from Pfizer Inc. The author reports no conflicts of interest in this work.

\section{References}

1. Wolfe F, Smythe HA, Yunus MB, et al. The American College of Rheumatology 1990 Criteria for the Classification of Fibromyalgia. Arthritis Rheum. 1990;33:160-172.

2. Neumann L, Buskila D. Epidemiology of fibromyalgia. Curr Pain Headache Rep. 2003;7:362-368.

3. Katz RS, Wolfe F, Michaud K. Fibromyalgia diagnosis: a comparison of clinical, survey, and American College of Rheumatology criteria. Arthritis Rheum. 2006;54(1):169-176.

4. Silverman S, Dukes EM, Johnson SS, Brandenburg NA, Sadosky A, Huse DM. The economic burden of fibromyalgia: comparative analysis with rheumatoid arthritis. Curr Med Res Opin. 2009;25(4):829-840.

5. Carville SF, Arendt-Nielsen S, Bliddal H, et al. EULAR evidence-based recommendations for the management of fibromyalgia syndrome. Ann Rheum Dis. 2008;67:536-541.

6. Scholz BA, Hammonds CL, Boomershine CS. Duloxetine for the management of fibromyalgia syndrome. J Pain Res. 2009;2:99-108.

7. Ormseth MJ, Eyler AE, Hammonds CL, Boomershine CS. Milnacipran for the management of fibromyalgia syndrome. J Pain Res. 2010; $3: 1-10$.

8. Pfizer.com [homepage on the Internet]. Pfizer, Inc.; c2002-2009 [updated 2009 Dec]. Available from: http://www.pfizer.com/files/ products/uspi_lyrica.pdf Accessed Mar 10, 2010.

9. Spaeth M. Is pregabalin a safe and effective treatment for patients with fibromyalgia? Nat Clin Pract Rheumatol. 2008;4:514-515.

10. Fink K, Dooley DJ, Meder WP, et al. Inhibition of neuronal $\mathrm{Ca}(2+)$ influx by gabapentin and pregabalin in the human neocortex. Neuropharmacology. 2002;42:229-236.

11. Russell IJ, Orr MD, Littman B, et al. Elevated cerebrospinal fluid levels of substance $\mathrm{P}$ in patients with the fibromyalgia syndrome. Arthrit Rheum. 1994;37(11):1593-1601.

12. Sarchielli P, Mancini ML, Floridi A, et al. Increased levels ofnNeurotrophinsa are not specific for chronic migraine: evidence from primary fibromyalgia syndrome. J Pain. 2007;8(9):737-745.

13. Baranauskas G, Nistri A. Sensitization of pain pathways in the spinal cord: cellular mechanisms. Prog Neurobiol. 1998;54:349-365.

14. Hauser W, Bernardy K, Uceyler N, Sommer C. Treatment of fibromyalgia syndrome with gabapentin and pregabalin. A meta-analysis of randomized controlled trials. Pain. 2009;145(1-2):69-81.

15. Crofford LJ, Rowbotham MC, Mease PJ, et al. Pregabalin for the treatment of fibromyalgia syndrome. Arthrit Rheum. 2005;52(4): $1264-1273$.

16. Farrar JT, Young JP Jr, Lamoreaux L, Werth JL, Poole RM. Clinical importance of changes in chronic pain intensity measured on an 11-point numerical pain rating scale. Pain. 2001;94:149-158.

17. Mease PJ, Russell IJ, Arnold LM, et al. A randomized, double-blind, placebo-controlled, phase III trial of pregabalin in the treatment of patients with fibromyalgia. $J$ Rheumatol. 2008;35(3):502-514. 
18. Dworkin RH, Turk DC, Farrar JT, et al. Core outcome measure for chronic pain clinical trials: IMMPACT recommendations. Pain. 2005; 113:9-19.

19. Burckhardt CS, Clark SR, Bennett RM. The fibromyalgia impact questionnaire (FIQ): development and validation. J Rheumatol. 1991; 18:728-733.

20. Crofford LJ, Mease PJ, Simpson SL, et al. Fibromyalgia relapse evaluation and efficacy for durability of meaningful relief (FREEDOM): a 6-month, double-blind, placebo-controlled trial with pregabalin. Pain. 2008;136:419-431.

21. Belza BL. Comparison of self-reported fatigue in rheumatoid arthritis and controls. J Rheumatol. 1995;22:639-643.

22. Hays RD, Stewart AL. In: Stewart AL, Ware JE, editors. Sleep Measures. Measuring Functioning and Well-being: The Medical Outcomes Study Approach. Durham, NC: Duke University Press; 1992:235-259.

23. Ware JE, Sherbourne CD. The MOS 36-item short-form health survey (SF-36): I. Conceptual framework and item selection. Med Care. 1992; 30:473-483.

24. Arnold LM, Russell IJ, Diri EW, et al. A 14-week, randomized, doubleblinded, placebo-controlled monotherapy trial of pregabalin in patients with fibromyalgia. J Pain. 2008;9(9):792-805.

25. Boomershine CS. First pregabalin and now duloxetine for fibromyalgia syndrome: closer to a brave new world? Nat Clin Pract Rheumatol. 2008;4:636-637.

26. Pauer L. Pregabalin for management of fibromyalgia (FM): a 14-week, randomized, double-blind, placebo-controlled monotherapy trial (study A0081100). 2008. Available from: http://www.clinicalstudyresults.org/ drugdetails/?drug_id=4636 Accessed Apr 2, 2010.

27. Zigmond AS, Snaith RP. The hospital anxiety and depression scale. Acta Psychiatr Scand. 1983;67:361-370.

28. Straube S, Derry S, Moore RA, McQuay HJ. Pregabalin in fibromyalgia: meta-analysis of efficacy and safety from company clinical trial reports. Rheumatology. 2010;49:706-715.

29. Zhang W, Jones A, Doherty M. Does paracetamol (acetaminophen) reduce the pain of osteoarthritis? A meta-analysis of randomised controlled trials. Ann Rheum Dis. 2004;63(8):901-907.
30. Sultan A, Gaskell H, Derry S, Moore RA. Duloxetine for painful diabetic neuropathy and fibromyalgia pain: systematic review of randomized trials. BMC Neurol. 2008;8:29.

31. Bennett RM, Kamin M, Karim R, Rosenthal N. Tramadol and acetaminophen combination tablets in the treatment of fibromyalgia pain: a double-blind, randomized, placebo-controlled study. Am J Med. 2003;114:537-545.

32. Boomershine CS, Crofford LJ. A symptom-based approach to pharmacologic management of fibromyalgia. Nat Rev Rheumatol. 2009;5(4): 191-199.

33. Eli Lilly and Company. Cymbalta presenbing information. 2004. Available from: http://pi.lilly.com/us/cymbalta-pi.pdf. Accessed Mar 19, 2010 .

34. Forest Pharmaceuticals. Savella ${ }^{\circledR}$ presenbing information. 2009. Available from: http://www.frx.com/pi/Savella_pi.pdf. Accessed Mar 29, 2010 .

35. US Department of Health and Human Services, Food and Drug Administration, Center for Drug Evaluation and Research, Office of Translational Sciences, Office of Biostatistics. Statistical Review and Evaluation: Antiepileptic Drugs and Suicidality. 2008 May. http://www.fda.gov/downloads/Drugs/DrugSafety/Postmarket DrugSafetyInformationforPatientsandProviders/UCM192556.pdf Accessed Mar 22, 2010.

36. Hindmarch I. A double-blind study in healthy volunteers to assess the effects on sleep of pregabalin compared with alprazolam and placebo. Sleep. 2005;28:187-193.

37. Hidalgo RB, Tupler LA, Davidson JRT. An effect-size analysis of pharmacologic treatments for generalized anxiety disorder. J Psychopharmacol. 2007;21:864-873.

38. Bech P. Dose-response relationship of pregabalin in patients with generalized anxeity disorder. Pharmacopsychiatry. 2007;40(4):163-168.

39. Raphael KG, Janal MN, Nayak S, Schwartz JE, Gallagher RM. Psychiatric comorbidities in a community sample of women with fibromyalgia. Pain. 2006;124:117-125.

40. Simpson DA. Gabapentin and venlafaxine for the treatment of painful diabetic neuropathy. J Clin Neuromuscul Dis. 2001;3:53-62.
Journal of Pain Research

\section{Publish your work in this journal}

The Journal of Pain Research is an international, peer-reviewed, open access, online journal that welcomes laboratory and clinical findings in the fields of pain research and the prevention and management of pain. Original research, reviews, symposium reports, hypothesis formation and commentaries are all considered for publication.

\section{Dovepress}

The manuscript management system is completely online and includes a very quick and fair peer-review system, which is all easy to use. Visit http://www.dovepress.com/testimonials.php to read real quotes from published authors. 